\title{
Bronchoscopy and endobronchial ultrasound for diagnosis and staging of lung cancer
}

\begin{abstract}
Various techniques, including standard bronchoscopy, transthoracic needle aspiration and mediastinoscopy, are used for diagnosis and staging of lung cancer. Minimizing the number of invasive procedures for lung cancer diagnosis and staging is preferred, however, and a growing number of bronchoscopic techniques are being used. Currently available techniques for the initial diagnosis of lung cancer include electromagnetic navigation bronchoscopy with computed tomography mapping and sample collection, endobronchial ultrasound (EBUS) using radial or convex probe tips, and the combination of the two approaches. EBUS with transbronchial needle aspiration (EBUS-TBNA) is highly specific and sensitive for the examination of mediastinal lymph nodes. Several studies have demonstrated the utility of this approach for less invasive lung cancer mediastinal staging. EBUS-TBNA has also been used in the collection of tissue samples for the analysis of tumor biomarkers that significantly influence the selection of cancer treatment strategies. Evidence suggests that EBUS-TBNA may be less useful for restaging patients with lung cancer after cytotoxic therapy.
\end{abstract}

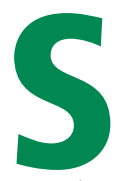
everal techniques are available for the diagnosis of suspected lung cancer, including standard flexible bronchoscopy, transthoracic needle aspiration, and sputum cytology. Mediastinal staging of lung cancer is essential for treatment planning and assessment of prognosis, and has traditionally been performed surgically. Although cervical mediastinoscopy is regarded as the "gold standard" for sampling mediastinal lymph nodes, this procedure typically requires hospitalization and gen-

Dr. Almeida reported that he has no financial relationships that pose a potential conflict of interest with this article.

This article was developed from an audio transcript of Dr. Almeida's presentation at the "Advances in Lung Cancer Evaluation and Management" symposium held in Cleveland, Ohio, on April 30, 2011. The transcript was formatted and edited by Cleveland Clinic Journal of Medicine staff for clarity and consciseness and was then reviewed, revised, and approved by Dr. Almeida.

doi:10.3949/ccjm.79.s2.03 eral anesthesia. ${ }^{1}$ Current endobronchial ultrasound (EBUS) techniques provide less invasive lung cancer diagnosis and staging. Recent research has examined the application of endobronchial ultrasound-based assessment for initial diagnosis of lung cancer, mediastinal staging and restaging after neoadjuvant therapy, and evaluation of tumor genetic markers.

\section{BRONCHOSCOPIC LUNG CANCER DIAGNOSIS}

Evidence-based clinical guidelines for the diagnosis of lung cancer developed by the American College of Chest Physicians reviewed the sensitivity of standard bronchoscopy (ie, without EBUS or electromagnetic navigation) and ancillary procedures that are often performed in combination with flexible bronchoscopy, such as endobronchial biopsy, brushing, washing, and standard transbronchial needle aspiration (TBNA). ${ }^{2}$ A comprehensive review of published studies from 1971 to 2004 was included in the analysis. Overall, the sensitivity of standard flexible bronchoscopy was $88 \%$ (67\% to $97 \%$ ) for the diagnosis of central bronchogenic carcinoma and 78\% (36\% to $88 \%$ ) for the diagnosis of peripheral bronchogenic carcinoma. Newer techniques have been developed that appear to provide more consistent diagnosis of primary lesions.

Electromagnetic navigation bronchoscopy (ENB) is a functional tool in biopsy planning that uses computed tomography (CT) mapping to precisely locate peripheral lesions. After real-time navigation to the peripheral lesion with a steerable probe, tissue collection may be optimized by guiding sampling instruments directly to the lesion through an extendable working channel. ${ }^{3}$ A prospective pilot study examined the feasibility and safety of ENB to reach peripheral lesions and lymph nodes in patients with suspected lung cancer lesions or enlarged mediastinal lymph nodes. ${ }^{3}$ Diagnostic tissue was obtained in $80.3 \%$ of attempts, including $74 \%$ of procedures involving peripheral lung lesions and $100 \%$ of procedures involving lymph nodes. 


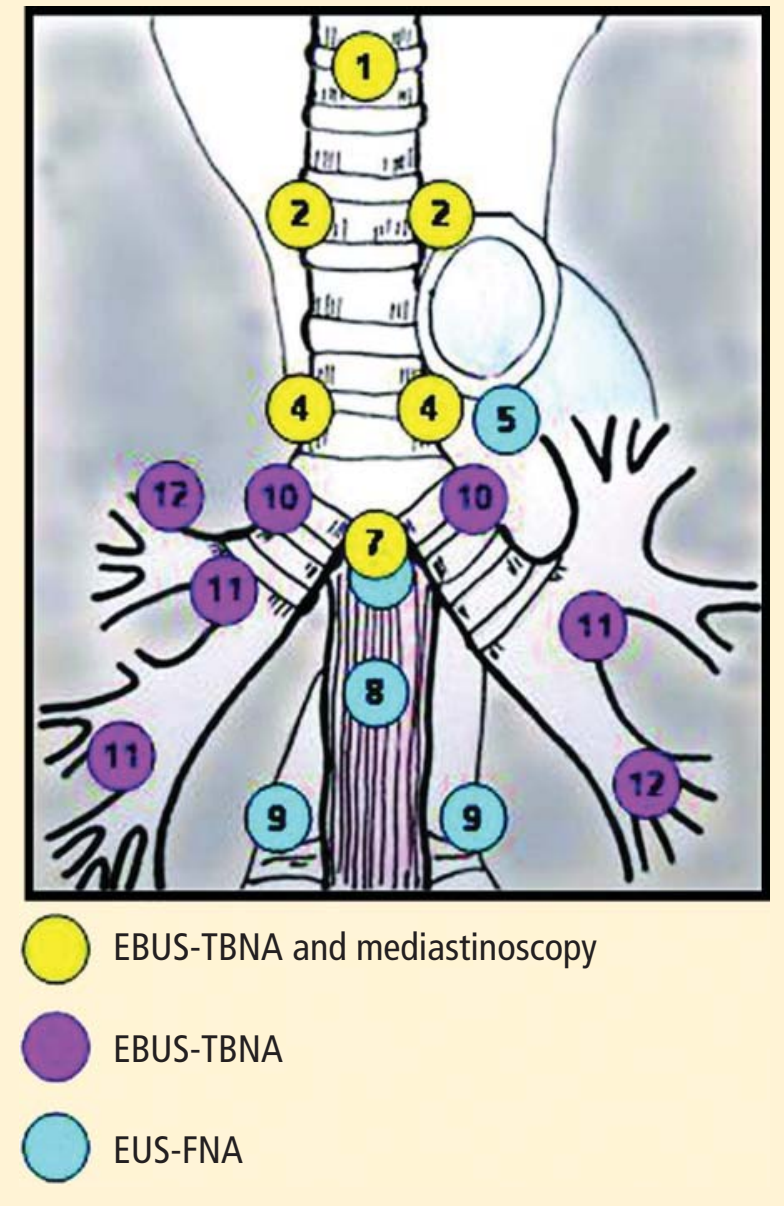

FIGURE 1. The diagnostic reach of various ultrasound sampling techniques is shown with 1 , highest mediastinal; 2 , upper paratracheal; 4, lower paratracheal; 5 , subaortic; 7 , subcarinal; 8 , paraesophageal; 9 , pulmonary ligament; 10 , hilar; 11 , interlobar; and 12, lobar. Endobronchial ultrasound with transbronchial needle aspiration (EBUS-TBNA) is performed via the airway as opposed to endoscopic ultrasound with fine-needle aspiration (EUS-FNA), which is carried out in the esophagus.?

Reproduced with permission from the American College of Chest Physicians (Yasufuku K, et al. Comparison of endobronchial ultrasound, positron emission tomography, and CT for lymph node staging of lung cancer. Chest 2006; 130:710-718).

\section{IMPROVING DIAGNOSIS WITH ULTRASOUND}

Another diagnostic method is EBUS, which uses reflected sound waves to better visualize lesions at the time of biopsy. ${ }^{4}$ Radial probe endobronchial ultrasound (RP-EBUS) employs a rotating ultrasound transducer at the end of a probe, and is used either with or without a water-filled balloon to improve ultrasound transduction and image quality. Convex-probe ultrasound uses a curvilinear ultrasound probe at the end of a bronchoscope, which allows for real-time TBNA visualization. ${ }^{4}$ A recent meta-analysis examined the yield of RP-EBUS for the evaluation of peripheral pulmonary lesions in 16 studies with a combined population of 1,420 patients. ${ }^{5}$ The overall sensitivity of RP-EBUS for the detection of lung cancer was $73 \%$, and the specificity was $100 \%$. In a prospective, randomized clinical trial of patients with peripheral lung lesions, the combination of ENB and RP-EBUS produced a diagnostic yield of $88 \%$, compared with 69\% with RP-EBUS alone and $59 \%$ with ENB alone $(P=.02) .6$ Although this finding suggests that a multimodal approach combining ENB and RP-EBUS may improve lung cancer diagnosis, the sample size was relatively small (118 patients).

\section{ENDOBRONCHIAL ULTRASOUND FOR LUNG CANCER STAGING}

A promising application for EBUS is its use as a less invasive method for confirming metastatic mediastinal lymph nodes in the staging of lung cancer. Figure 1 shows the distribution of the mediastinal lymph nodes and the various diagnostic techniques that may be used to sample different lymph node stations. ${ }^{?}$

In a prospective study of potentially operable patients from Japan with proven $(\mathrm{n}=96)$ or suspected $(\mathrm{n}=6)$ lung cancer, investigators compared CT, positron emission tomography (PET), and EBUS-TBNA for mediastinal lymph node staging using surgical histology as the reference standard. ${ }^{7}$ The accuracy of staging was significantly greater with EBUS-TBNA (98\%) than either PET $(72.5 \%)$ or CT $(60.8 \%)(P<.00001)$.

A recent retrospective study examined the use of EBUS-TBNA for clarification of 127 PET-positive hilar or mediastinal lymph nodes from 109 patients with suspected lung cancer. ${ }^{1}$ In 77 patients (71\%), EBUS-TBNA successfully identified cancerous lymph nodes and obviated the need for further surgical biopsy. In 96 patients with definitive reference pathology, the sensitivity of EBUS-TBNA was $91 \%$, specificity was $100 \%$, and diagnostic accuracy was $92 \%$. The positive predictive value of EBUS was $100 \%$, but the negative predictive value (ie, the proportion of patients with negative EBUS-TBNA who were also negative on surgical pathology) was only $60 \%$. This suggests a relatively high rate of false-negative EBUS-TBNA findings in this PET-positive group of patients.

Another recent study prospectively evaluated the usefulness of EBUS-TBNA after PET-CT for mediastinal staging in 117 patients with potentially operable non-small cell lung cancer (NSCLC). ${ }^{8}$ Patients were classified as either N2- or N3-positive or -negative using EBUS-TBNA, and patients who were N2- or N3-negative underwent surgical staging with lymph node dissection. Mediastinal node metastasis was 
confirmed by EBUS-TBNA in 37 nodal stations of 27 patients. Ninety patients who were negative by EBUS-TBNA underwent surgery with lymph node dissection. Three were reclassified as positive and 87 as negative. The overall sensitivity of EBUS-TBNA for the detection of mediastinal metastases was 90\% versus $70 \%$ with PET-CT $(P=.052)$. For the subgroup of 61 patients who had a normal mediastinum by PET-CT, nine were found to have mediastinal metastases at surgical evaluation. Six of these nine falsenegatives were correctly identified by EBUS-TBNA.

Similar results were found in a study examining the use of EBUS-TBNA in 97 patients with confirmed NSCLC, no enlarged lymph nodes on CT (ie, no lymph nodes larger than $1 \mathrm{~cm}$ in short axis), and no abnormal mediastinal PET findings. ${ }^{9}$ Lymph nodes as small as $5 \mathrm{~mm}$ by ultrasound imaging at stations $2 \mathrm{R}$, 2L, 4R, 4L, 7, 10R, 10L, 11R, and 11L were aspirated, and all patients underwent surgical staging. Malignant lymph nodes were detected by surgical staging in nine patients, and eight of these were identified by EBUSTBNA. The sensitivity of EBUS-TBNA for the detection of mediastinal metastases was $89 \%$; the specificity was $100 \%$; and the negative predictive value was $99 \%$.

\section{Guided fine-needle aspiration with ultrasound bronchoscopy}

An additional approach to mediastinal lung cancer staging is endoscopic ultrasound with bronchoscope-guided fine-needle aspiration (EUS-B-FNA) and EBUSTBNA in a single procedure. The use of EBUS-TBNA and EUS-B-FNA for NSCLC staging was examined in a prospective study of 150 patients with confirmed or strongly suspected NSCLC. ${ }^{10}$ Patients underwent EBUS-TBNA, and EUS-B-FNA then was used for nodes that were inaccessible through the airways. EBUS-TBNA diagnosed mediastinal metastases in 38 of 143 patients, and three more patients were identified by additional EUS-B-FNA. Surgery identified four additional patients with mediastinal metastases that were negative by both EBUS-TBNA and EUS-B-FNA. Overall sensitivity for the detection of mediastinal metastases was $84.4 \%$ with EBUS-TBNA alone versus 91.1\% with EBUS-TBNA followed by EUS-B-FNA, but this was not statistically significant $(P=.332)$.

A second study of 139 patients with confirmed NSCLC reported similar results when EBUS-TBNA and EUS-B-FNA were performed using a single ultrasound bronchoscope. ${ }^{11}$ The sensitivity for detection of mediastinal metastases was $89 \%$ with EUS-FNA, 92\% with EBUS-TBNA, and 96\% with the combined approach. The specificity was 100\% for all three approaches. The negative predictive values were $82 \%$ for the esophageal approach, 92\% for the endobronchial approach, and $86 \%$ for the combined approach.

\section{Meta-analyses support EBUS-TBNA for staging}

The usefulness of EBUS-TBNA for NSCLC staging has been examined in two recent meta-analyses. The first included data from 11 studies of EBUS-TBNA with 1,299 patients. ${ }^{12}$ Overall, the included studies yielded a pooled sensitivity of $93 \%$ and a specificity of $100 \%$ for the detection of metastatic mediastinal lymph nodes $(95 \% \mathrm{CI})$. The sensitivity was higher for patients who were selected for evaluation on the basis of positive PET or CT findings than for patients without selection by PET or CT $(0.94$ vs 0.76$)(P<.05)$. The authors concluded that EBUS-TBNA for lung cancer staging is accurate, safe, and cost-effective, and that selection of patients based on CT or PET findings resulted in higher sensitivity.

The second meta-analysis examined data from 10 studies evaluating the utility of EBUS-TBNA for lung cancer staging. ${ }^{13}$ This meta-analysis also yielded high sensitivity (88\%) and specificity (100\%) of EBUS-TBNA for the identification of metastatic mediastinal lymph nodes.

\section{EVALUATION OF EBUS VERSUS MEDIASTINOSCOPY AND OTHER INVASIVE TESTS}

Although several studies suggest that EBUS-TBNA provides an accurate and less invasive method for assessment of mediastinal lymph nodes in the mediastinal staging of patients with NSCLC, few studies have directly compared EBUS-TBNA with mediastinoscopy. In a prospective crossover trial, 66 patients with suspected NSCLC underwent mediastinal staging using EBUS-TBNA followed by mediastinoscopy, with surgical lymph node dissection as the reference standard. ${ }^{14}$ The overall diagnostic yield for all lymph nodes was significantly higher with EBUS-TBNA than with mediastinoscopy $(91 \%$ vs $78 \%)(P=.007)$. However, this difference was primarily due to a higher success rate in the diagnosis of subcarinal lymph nodes (98\% vs $78 \%)(P=.007)$, which can be difficult to evaluate with mediastinoscopy. Differences between the two methods at other node stations were not statistically significant (Table). In the 57 patients who were diagnosed with NSCLC, the prediction of the correct pathologic stage did not differ significantly between the two approaches (93\% with EBUS-TBNA vs $82 \%$ with mediastinoscopy) $(P=.083)$.

A more recent randomized, multicenter clinical trial compared endosonographical staging (EUS- 
TABLE

Diagnostic yield of EBUS-TBNA and mediastinoscopy is significant in the elevation for all lymph nodes, but varies across the lymph node stations

$\begin{array}{lcccc} & \begin{array}{c}\text { Lymph node size in mm } \\ \text { mean } \pm \text { SD (range) }\end{array} & \begin{array}{c}\text { Mediastinoscopy } \\ \text { yield (\%) }\end{array} & \text { pa } \\ \text { All lymph nodes } & 15 \pm 2.6(10-21) & 109 / 120(91) & 94 / 120(78) & .007 \\ \text { Lymph node station } & & & & \\ 2 \text { all } & 16 \pm 3.1(10-21) & 24 / 25(96) & 22 / 25(88) & .30 \\ 2 \text { right } & 18 \pm 1.6(14-20) & 12 / 13(92) & 11 / 13(85) & .99 \\ 2 \text { left } & 14 \pm 3.6(10-21) & 12 / 12(100) & 11 / 12(92) & .99 \\ 4 \text { all } & 15 \pm 2.6(10-19) & 45 / 54(83) & 40 / 54(74) & .24 \\ 4 \text { right } & 15 \pm 2.6(10-19) & 29 / 34(85) & 24 / 34(71) & .14 \\ 4 \text { left } & 15 \pm 2.6(10-19) & 16 / 20(80) & 16 / 20(80) & .99 \\ 7 & 15 \pm 2.4(10-19) & 40 / 41(98) & 32 / 41(78) & .007 \\ \end{array}$

a $P$ value by chi square test.

EBUS-TNA = endobronchial ultrasound-guided transbronchial needle aspiration

Reprinted with permission from Journal of Thoracic Oncology (Ernst A, et al. Diagnosis of mediastinal adenopathy-real-time endobronchial ultrasound guided needle aspiration versus mediastinoscopy. J Thorac Oncol 2008; 3:577-582).

examination, the patient should undergo CT-PET or chest CT with contrast that also should assess the liver and adrenal glands. If the patient has radiographic evidence of metastatic disease, the next step is biopsy of the most accessible, most advanced lesion for tissue diagnosis and staging. In patients without evidence of metastatic disease, the next step is to evaluate the mediastinal lymph nodes. Patients with evidence of nodal involvement on PETCT or without evidence of nodal involvement but with larger tumors (eg, stage $\mathrm{T} 1 \mathrm{~b}$ or larger) may be evaluated using EBUS-

FNA and EBUS-TBNA) with mediastinoscopy in 241 patients with resectable suspected NSCLC. ${ }^{15}$ Patients were randomized to either surgical staging or to endosonography followed by surgical staging for those without nodal metastases using ultrasoundguided FNA. The sensitivity for detection of nodal metastases was $79 \%$ with surgical staging and $94 \%$ with endosonography and surgical staging $(P=.02)$. Comparing the sensitivity of the two procedures alone, without follow-up surgical staging when ultrasound was negative, the sensitivities of the two approaches were similar: $79 \%$ with mediastinoscopy and $85 \%$ with endosonographic staging alone.

Another retrospective study examined the results of EBUS-TBNA for the initial diagnosis and staging of 88 patients with known or suspected lung cancer who underwent at least one invasive diagnostic or staging procedure before EBUS-TBNA. ${ }^{16}$ The selection of EBUS-TBNA and bronchoscopy as the initial test for diagnosis and staging could have prevented at least one invasive test in $50 \%$ of patients, and could have been the only invasive test procedure in $47.7 \%$ of individuals. In 27 patients who underwent two or more invasive tests, EBUS-TBNA could have avoided at least one invasive test in 16 patients (59\%).

\section{PATHWAYS TO DIAGNOSIS}

A proposed diagnostic algorithm for suspected NSCLC is shown in Figure 2. ${ }^{17}$ When lung cancer is highly suspected on the basis of focused patient history and physical
TBNA as the first invasive test if available or mediastinoscopy. Standard bronchoscopy in conjunction with EBUS-TBNA has the capability of sampling the primary lesion when the mediastinal staging fails to demonstrate malignant disease. Therefore, it can provide a definitive diagnosis in addition to mediastinal staging during one single procedure, whereas mediastinoscopy typically cannot assess the primary lesion if necessary.

\section{APPLICATIONS IN MOLECULAR TUMOR PROFILING}

Genetic profiling of lung cancer tissue samples is essential to identify biomarkers that significantly influence treatment responses, and EBUS-TBNA has been used to obtain biopsy tissue samples for genetic analysis. One study examined the detection of EGFR gene mutations in biopsy tissue samples obtained from 46 patients with metastatic adenocarcinoma to the hilar or mediastinal lymph nodes diagnosed by EBUS-TBNA. ${ }^{18}$ Recut sections of the paraffin-embedded samples yielded tumor cells in 43 patients, and tissue samples were examined for mutations of EGFR exons 19 and 21. Five patients underwent surgical resection, and three of these yielded samples with EGFR mutations at exon 21. Examination of the 43 EBUS-TBNA specimens revealed EGFR mutations in 11 . These included three of the mutations that were identified from surgical specimens. A more recent study examined the concordance between mutations of KRAS, EGFR, BRAF, and PIK3CA obtained by EBUS-TBNA, EUS-B-FNA, and histologic samples obtained during surgical staging from 


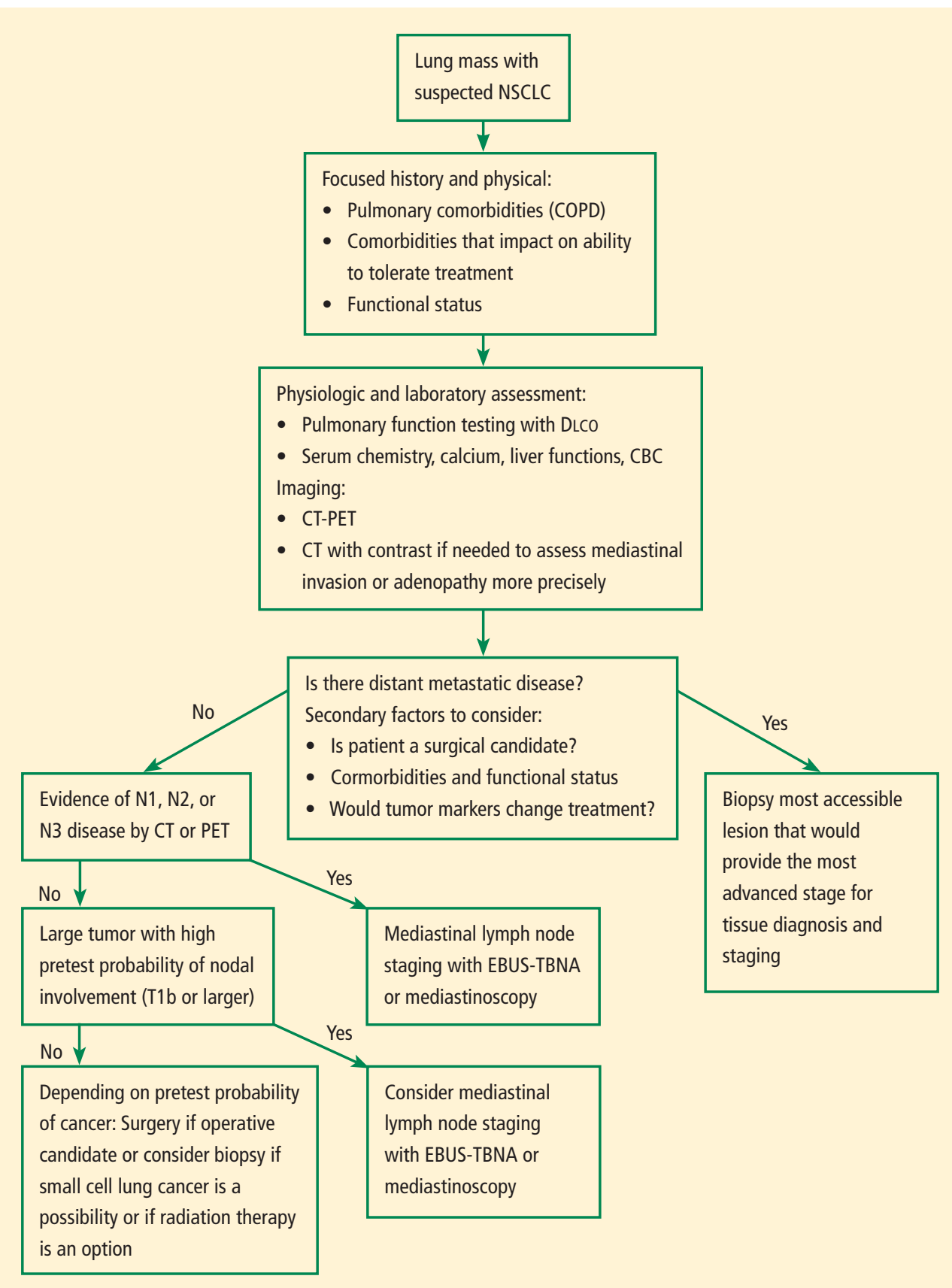

FIGURE 2. This diagnostic algorithm should be followed for patients with suspected non-small cell lung cancer (NSCLC). ${ }^{17} \mathrm{CBC}=$ complete blood count; $\mathrm{COPD}=$ chronic obstructive pulmonary disease; $\mathrm{CT}=$ computed tomography; DLCO = diffusion capacity of the lung for carbon monoxide; EBUS-TBNA = endobronchial ultrasound-guided transbronchial needle aspiration; PET = positron emission tomography

Reprinted with permission from Current Opinion in Pulmonary Medicine (Almeida FA, et al. Initial evaluation of the nonsmall cell lung cancer patient: diagnosis and staging. Curr Opin Pulm Med 2010; 16:307-314).

43 patients. ${ }^{19}$ KRAS mutations were identified in six patients, EGFR mutation in one patient, and PIK3CA mutation in one patient. The investigators observed $100 \%$ concordance between cytologic fine-needle aspirates and histologic specimens, suggesting no additional benefit of more invasive procedures for the evaluation of tumor biomarkers.

\section{EBUS RESTAGING OF LUNG CANCER}

The utility of EBUSTBNA has also been investigated for restaging of lung cancer following neoadjuvant chemotherapy. Mediastinal restaging using EBUS-TBNA was performed in 124 consecutive patients with stage IIIA-N2 NSCLC who had received chemotherapy induction. ${ }^{20}$ CT evaluation revealed partial responses for 66 patients and stable disease in 58. All patients subsequently underwent thoracotomy and attempted curative resection with lymph node dissection. Of 58 patients with stable disease on CT, 41 were EBUS-TBNA-positive for mediastinal metastasis, and all were thoracotomypositive. However, in 17 patients who were EBUSTBNA-negative, 14 were thoracotomy-positive and only three were thoracotomy-negative. Similarly, in 66 patients with partial response to treatment on CT, 48 were EBUSTNA-positive and thoracotomy-positive. In 18 patients who were EBUSTBNA-negative, 14 were thoracotomy-positive and only four were also thoracotomy-negative. Overall, the sensitivity of EBUS-TBNA was $77 \%$ in patients with partial responses and $75 \%$ in those with stable disease. The negative predictive value of EBUS-TBNA in this 
series was very low: $22 \%$ in the partial response group and $18 \%$ in the stable disease group.

Similar results were obtained in a European study that examined EBUS-TBNA mediastinal restaging after neoadjuvant therapy in patients with pathologically confirmed N2 disease. ${ }^{21}$ Patients with negative or uncertain EBUS-TBNA were reexamined using transcervical extended bilateral mediastinal lymphadenectomy, a surgical staging procedure that is not widely used in the United States. Of 85 mediastinal lymph nodes from 61 patients that were examined using EBUS-TBNA, nine patients (15\%) had a falsenegative result with EBUS-TBNA, and three patients $(5 \%)$ had a false-positive result. On a per-patient basis, the sensitivity of EBUS-TBNA was $67 \%$ and the negative predictive value was $78 \%$.

\section{SUMMARY AND CONCLUSIONS}

Newer technologies such as EBUS-TBNA make it possible to simplify the diagnosis and staging of lung cancer. Bronchoscopy with EBUS may be the preferred method for the initial diagnosis and staging of patients who have disease limited to the chest. EBUS is clearly superior to current modalities for mediastinum staging such as CT and PET, and appears to be similar to mediastinoscopy. Standard bronchoscopy with EBUS followed by mediastinoscopy, if necessary, appears to be the best strategy for initial diagnosis and staging of patients with suspected lung cancer radiographically limited to the chest. However, at this time, diagnosis and staging should rely on local expertise rather than a particular methodology. Patients with T1B lesions or higher should be considered for invasive mediastinal staging regardless of their PET or CT results. The available evidence suggests that EBUS is a reasonable initial test for mediastinal restaging following neoadjuvant chemotherapy. However, a negative EBUS in this setting should prompt additional invasive tests to confirm its findings.

\section{REFERENCES}

1. Rintoul RC, Tournoy KG, El Daly H, et al. EBUS-TBNA for the clarification of PET positive intra-thoracic lymph nodes: an international multi-centre experience. J Thorac Oncol 2009; 4:44-48.

2. Rivera P, Metha A, American College of Chest Physicians. Initial diagnosis of lung cancer: ACCP evidence-based clinical practice guidelines (2nd edition). Chest 2007; 132:131S-148S.

3. Gildea TR, Mazzone PJ, Karnak D, Meziane M, Mehta AC. Electromagnetic navigation diagnostic bronchoscopy: a prospective study. Am J Respir Crit Care Med 2006; 174:982-989.

4. Sheski FD, Mathur PN. Endobronchial ultrasound. Chest 2008; 133:264-270

5. Steinfort DP, Khor YH, Manser RL, Irving LB. Radial probe endobronchial ultrasound for the diagnosis of peripheral lung cancer: systematic review and meta-analysis. Eur Respir J 2011; 37:902-910.

6. Eberhardt R, Anantham D, Ernst A, Feller-Kopman D, Herth F.
Multimodality bronchoscopic diagnosis of peripheral lung lesions: a randomized controlled trial. Am J Respir Crit Care Med 2007; 176:36-41.

7. Yasufuku K, Nakajima T, Motoori K, et al. Comparison of endobronchial ultrasound, positron emission tomography, and CT for lymph node staging of lung cancer. Chest 2006; 130:710-718.

8. Hwangbo B, Kim SK, Lee HS, et al. Application of endobronchial ultrasound-guided transbronchial needle aspiration following integrated $\mathrm{PET} / \mathrm{CT}$ in mediastinal staging of potentially operable non-small cell lung cancer. Chest 2009; 135:1280-1287.

9. Herth FJ, Eberhardt R, Krasnik M, Ernst A. Endobronchial ultrasound-guided transbronchial needle aspiration of lymph nodes in the radiologically and positron emission tomography-normal mediastinum in patients with lung cancer. Chest 2008; 133:887-891.

10. Hwangbo B, Lee GK, Lee HS, et al. Transbronchial and transesophageal fine-needle aspiration using an ultrasound bronchoscope in mediastinal staging of potentially operable lung cancer. Chest 2010; 138:795-802.

11. Herth FJ, Krasnik M, Kahn N, Eberhardt R, Ernst A. Combined endoscopic-endobronchial ultrasound-guided fine-needle aspiration of mediastinal lymph nodes through a single bronchoscope in 150 patients with suspected lung cancer. Chest 2010; 138:790-794.

12. Gu P, Zhao YZ, Jiang LY, Zhang W, Xin Y, Han BH. Endobronchial ultrasound-guided transbronchial needle aspiration for staging of lung cancer: a systematic review and meta-analysis [published online ahead of print January 3, 3009]. Eur J Cancer 2009; 45:1389_ 1396. doi:10.1016/j.ejca2009.06.023

13. Adams K, Shah PL, Edmonds L, Lim E. Test performance of endobronchial ultrasound and transbronchial needle aspiration biopsy for mediastinal staging in patients with lung cancer: systematic review and meta-analysis [published online ahead of print May 18, 2009]. Thorax 2009; 64:757-762. doi:10.1136/thx.2008.109868

14. Ernst A, Anantham D, Eberhardt R, Krasnik M, Herth FJ. Diagnosis of mediastinal adenopathy-real-time endobronchial ultrasound guided needle aspiration versus mediastinoscopy. J Thorac Oncol 2008; 3:577-582.

15. Annema JT, van Meerbeeck JP, Rintoul RC, et al. Mediastinoscopy vs endosonography for mediastinal nodal staging of lung cancer: a randomized trial. JAMA 2010; 304:2245-2252.

16. Almeida FA, Uzbeck M, Jimenez C, et al. Flexible bronchoscopy and endobronchial ultrasound-transbronchial needle aspiration (EBUS-TBNA) vs other invasive modalities in the initial diagnosis and staging of suspected or confirmed lung cancer. Chest 2010; 138:423A. Abstract.

17. Almeida FA, Uzbeck M, Ost D. Initial evaluation of the nonsmall cell lung cancer patient: diagnosis and staging. Curr Opin Pulm Med 2010; 16:307-314.

18. Nakajima T, Yasufuku K, Suzuki M, et al. Assessment of epidermal growth factor receptor mutation by endobronchial ultrasound-guided transbronchial needle aspiration [published online ahead of print June 15, 2007. Chest 2007; 132:597-602. doi:10.1378/chest.07-0095

19. van Eijk R, Licht J, Schrumpf M, et al. Rapid KRAS, EGFR, BRAF and PIK3CA mutation analysis of fine needle aspirates from non-small-cell lung cancer using allele-specific qPCR. PLoS One 2011; 6:e17791.

20. Herth FJ, Annema JT, Eberhardt R, et al. Endobronchial ultrasound with transbronchial needle aspiration for restaging the mediastinum in lung cancer [published online ahead of print June 2, 2008]. J Clin Oncol 2008; 26:3346-3350. doi:10.1200/JCO.2007.14.9229

21. Szlubowski A, Herth FJ, Soja J, et al. Endobronchial ultrasoundguided needle aspiration in non-small-cell lung cancer restaging verified by the transcervical bilateral extended mediastinal lymphadenectomy - a prospective study [published online ahead of print December 22, 2009]. Eur J Cardiothorac Surg 2010; 37:1180-1184. doi:10.1016/j.ejcts.2009.11.014

Correspondence: Francisco Aécio Almeida, MD, MS, FCCP, Critical Care Medicine, Cleveland Clinic, 9500 Euclid Avenue, A90, Cleveland, OH 44195; almeidf@ccf.org 